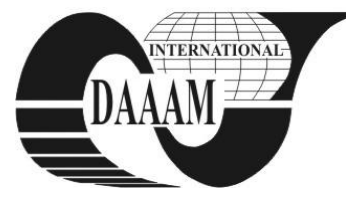

\title{
ON THE NOISE OF MAGNETIC MICROSENSORS IN BIPOLAR TECHNOLOGY
}

\author{
CARUNTU, G[eorge]; PANAIT, C[ornel] \& DUMITRASCU, A[na]
}

\begin{abstract}
The optimal processing of sensors - provided signal, imposes their integration on the same chip with the amplifier circuit. This paperwork analyse the structure, the operating conditions and the main features of some microsensors realised in bipolar integrated circuits technology. The electrical diagrams of the transducers which contain such sensors are also presented a number of conclusions are highlighted.

Key words: the transverse hall current, supply-current-related sensitivity, noise equivalent magnetic induction, shot noise, noise spectral density
\end{abstract}

\section{INTRODUCTION}

This paper presents a novel magnetotransistor structure based on the model of dual Hall devices. The operation conditions are analysed and the noise main characteristic of the structure realised in the bipolar technology are established.

The noise-equivalent magnetic induction is usually defined for conventional Hall devices, without the existing of any studies, regarding optimization of each value.

In this paper the values of the noise-equivalent magnetic induction are determined by means of numerical simulation for different structure devices and compared with values from literature. It is also emphasized the device performances dependency on the geometry and on the material properties.

In the final part the electrical diagrams of the transducers which contain such sensors are presented and described.

\section{GENERAL CHARACTERISATION OF THE SPLIT-COLLECTOR MAGNETOTRANSISTOR}

Figure 1 illustrates the cross section of a split-collector magnetotransistor operating on the current deflection principle (Drăgulinescu, 2005).

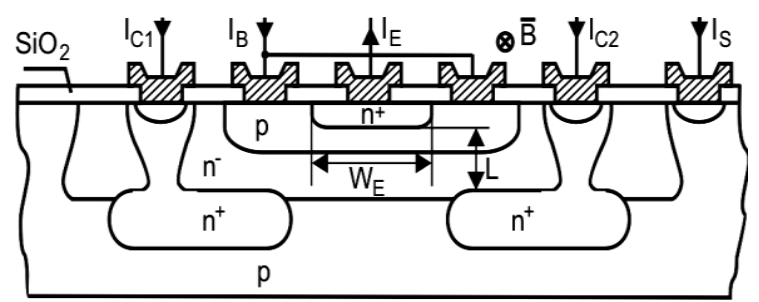

Fig. 1. The structure of a double-collector magnetotransistor

This structure is compatible with bipolar integrated circuits technology. In the absence of a magnetic field the electrons flow injected from the emitter which crosses the base is symmetrical and the collector currents are equal, $I_{C 1}=I_{C 2}$.

In the presence of a magnetic field with induction $B_{\perp}$, the distribution of the emitter electrons current is asymmetrical and causes an imbalance in the two collector currents, $\Delta I_{C}=I_{C 1}-I_{C 2}$. Since the output signal of the double collector magnetotransistor consists of the current variation between its terminals this device operates in the Hall current mode. Assimilating the low-doped epitaxial layer of the collector region with a short Hall plate, and based on the properties of dual Hall devices it results (Căruntu, 2009)

$$
\Delta I_{C}=\frac{I_{H}}{2}=\frac{1}{2} \mu_{H n} \frac{L}{W_{E}} G \cdot I_{C} B_{\perp}
$$

where $\mu_{H n}$ denotes the carriers Hall mobility, $G$ is the geometrical correction factor, $L$ is the emitter-collector distance, $W_{E}$ is the emitter with (see figure 1) and $I_{C}=I_{C 1}(0)+I_{C 2}(0)$.

\section{THE SENSITIVITY AND NOISE-EQUIVALENT MAGNETIC INDUCTION}

A magnetotransistor may be regarded as a modulation transducer that converts the magnetic induction signal into an electric current signal.

The output current signal is generated by the variation of collector current, caused by induction $B_{\perp}$. The supply-current related sensitivity of the device is defined by

$$
S_{I}=\frac{1}{I_{C}}\left|\Delta I_{C} / B_{\perp}\right|=\frac{1}{2} \mu_{H n}\left(L / W_{E}\right) G
$$

The noise current at the output of a magnetotransistor can be interpreted as a result of an equivalent magnetic induction. The mean square value of noise-equivalent magnetic induction ()NEMI is defined by (Popović, 1991):

$$
\left\langle B_{N}^{2}\right\rangle=\left(\int_{f_{1}}^{f_{2}} S_{N I}(f) \cdot d f\right) /\left(S_{I} \cdot I_{C}\right)^{2}
$$

where $S_{N I}$ is the noise current spectral density in the collector current, and $\left(f_{1}, f_{2}\right)$ is the frequency range.

In case of shot noise, the noise current spectral density at frequencies over $100 \mathrm{~Hz}$ is given by :

$$
S_{N I}=2 q I
$$

where $I$ is the device current

In a narrow frequency band around the frequency $f$, by substituting (2) and (4) into (3) results:

$$
<B_{N}^{2}>\leq 8 q\left(\frac{W_{E}}{L}\right)^{2} \cdot \frac{\Delta f}{G^{2}} \cdot \frac{1}{\mu_{H n}^{2}} \cdot \frac{1}{I_{C}}
$$

Considering that the condition of low value magnetic field are fulfilled $\left(\mu_{H}^{2} B^{2}<<1\right)$, a maximum value for 
$\left(L / W_{E}\right) G=0.74, \quad$ if $\quad W_{E} / L<0.5 \quad$ it $\quad$ is $\quad$ obtained (Middelhoek, 1989). In this case:

$$
<B_{N}^{2}>_{\min } \leq 14.6 q \frac{\Delta f}{I_{C}} \cdot \frac{1}{\mu_{H n}^{2}}
$$

The NEMI values obtained by simulation of three magnetotransistors structures built up from different materials are shown in figure 2.

$$
\begin{aligned}
& M G T_{1} \text { : Si with } \mu_{H n}=0.15 m^{2} V^{-1} s^{-1} \\
& M G T_{2}: \text { InP with } \mu_{H n}=0.46 m^{2} V^{-1} s^{-1} \\
& M G T_{3} \text { : GaAs with } \mu_{H n}=0.85 m^{2} V^{-1} s^{-1}
\end{aligned}
$$

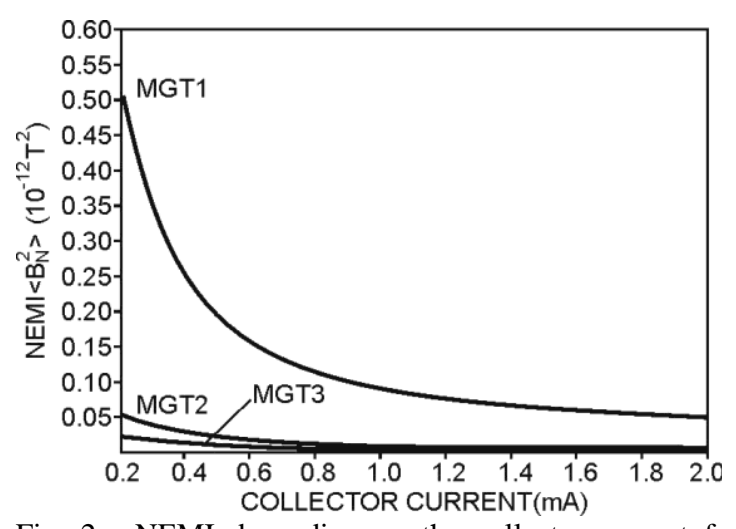

Fig. 2. NEMI depending on the collector current for three devices of different materials

To emphasize the dependence of NEMI on device geometry three double-collector magnetotransistors structures realised on silicon with $\mu_{H n}=0.15 m^{2} V^{-1} s^{-1}$, and having different ratios $W_{E} / L\left(W_{E}=50 \mu m\right)$ were simulated. The results obtained are shown in figure 3 . The devices are operating in the linear region and the magnetic field has a very low level $\left(\mu_{H}^{2} B^{2}<<1\right)$.

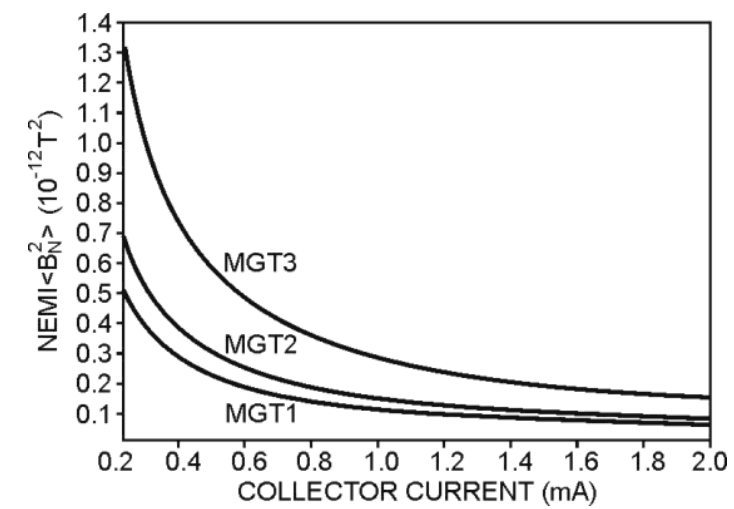

Fig. 3. NEMI depending on the collector current for three devices of different geometry

$$
\begin{aligned}
& M G T_{1} \text { with } W_{E} / L=0.5 \text { and }\left(L G / W_{E}\right)^{2}=0.576 \\
& M G T_{2} \text { with } W_{E} / L=1.0 \text { and }\left(L G / W_{E}\right)^{2}=0.409 \\
& M G T_{3} \text { with } W_{E} / L=02 \text { and }\left(L G / W_{E}\right)^{2}=0.212
\end{aligned}
$$

From those results we can observe that the NEMI is minimum for $W_{E} / L=0.5$, and for smaller values of this ratio. The decreasing of the channel length causes the increasing of
NEMI with $40.8 \%$ for a square structure $W_{E}=L$ and with $173 \%$ for $W=2 L$.

\section{CONCLUSIONS}

The analise of the characteristics of magneto-treansistor structures shows that the $W / L=0.5$ ratio is theoretically favourable to achieve high performance regarding the noise equivalent magnetic induction.

The noise equivalent magnetic induction lowers with the increase of carriers mobility, this increase being significant for collector currents of relatively low values. So for the collector current $I_{C}=0.3 \mathrm{~mA}$, the noise equivalent magnetic induction value of the GaAs device decreases by $88.6 \%$ as compared to that of the silicon device.

The use of magnetotransistors as magnetic sensors allows achieving of some current-voltage conversion circuits, more efficient than conventional circuits with Hall plates.

In figure 4 is shown the electrical diagram of a speed of rotation transducers based on a double-collector vertical magnetotransistors (Panait, 2007).

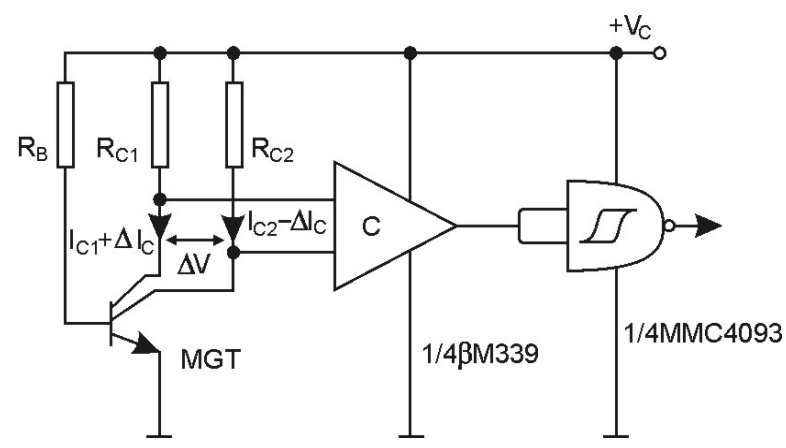

Fig. 4. The electric diagram of transducer

When a magnetic field is present an imbalance of the collector currents appears and the effect is potential difference between the two collectors which is proportional to the induction value $B_{\perp}$.

$$
\Delta V_{C}=\mu_{H n}\left(L / W_{E}\right) G R_{C} I_{C} B_{\perp}
$$

This voltage is applied to a comparator with hysteresis, which acts as a switch. The existence of the two travel thresholds ensure the immunity at noise to the circuit. The monostable made with $M M C 4093$ ensures the same duration for the transducers generated pulses.

\section{REFERENCES}

Căruntu, G. (2009). The Optimization of Bipolar Magnetotransistor Structures, Proceedings of SPIEVolume 7297 (2009)., pp. 72972M-72972M-6 (2009).

Drăgulinescu, M. (2005) The noise equivalent magnetic induction spectral density of magnetotransistors, Proceedings of CAS 2005, October 3,5 Romania, ISBN 07803-9214-0, pp 451-454, Sinaia

Middelhoek, S.; Audet, S.A.(1989). Physics of Silicon Sensors, pp. 5.20-5.24, Academic Press, ISBN 0124950511, London

Panait, C. (2007) The magnetic microsensors response, Przeglad Elektrotechniczny, Nr2/2007, pp 33-77, ISSN1731-6106 R, Poland

Popovic, R.S. (1991). Hall Effect Devices, Magnetic Sensors and Characterization of Semiconductors, Adam Hilger, ISBN 0750300965, Bristol, England 